\title{
Rechtspopulismus in der EU - Bedrohung für den Integrationsprozess
}

Der schon bei den letzten Wahlen zum Europaparlament (EP) im Jahre $2014 \mathrm{zu}$ beobachtende Rechtsruck hat sich seitdem durch Erfolge rechtspopulistischer Parteien in vielen Staaten der Europäischen Union (EU) weiter verfestigt. Der Beitrag untersucht die Ursachen eines anwachsenden Rechtspopulismus in ausgewählten Mitgliedstaaten sowie die weitreichenden Folgen der daraus erwachsenen politischen Instabilität. Es wird gezeigt, dass die EU aufgrund der zunehmenden ReNationalisierung nicht mehr im ausreichenden Maße in der Lage ist, für wichtige Konflikte eine Lösung zu finden. Das gilt u. a. für die Frage einer obligatorischen Verteilung von Flüchtlingen im Rahmen der Migrationspolitik wie auch für die Umsetzung wichtiger Reformvorschläge zur Stabilisierung der Eurozone.

KLAUS BUSCH

1 Ursachen des anwachsenden Rechtspopulismus in wichtigen EU-Staaten

In den letzten beiden Jahren haben in verschiedenen EU-Staaten, den Niederlanden, Frankreich, Österreich, Deutschland, Italien, Ungarn und Schweden, Parlamentswahlen stattgefunden. Bei diesen Wahlen haben die rechtspopulistischen Parteien deutliche Zugewinne verzeichnet. In Österreich ist seitdem die FPÖ an der Regierung beteiligt, in Italien stellen jetzt zwei populistische Parteien (Lega und Movimento Cinque Stelle) die Regierung und in Ungarn hat die Orbán-Partei Fidesz ihre Macht behauptet. Man kann mit Fug und Recht von einer deutlichen Rechtsverschiebung des politischen Spektrums in der EU sprechen.

Vergleichende Untersuchungen legen nahe, dass für die Ausbreitung des Rechtspopulismus insgesamt mehrere Faktoren ursächlich sind (Busch et al. 2018). Fünf Einflussgrößen können dabei als besonders relevant hervorgehoben werden: die ökonomische Entwicklung des Landes, die Entwicklung der sozialen Ungleichheit und deren Wahrnehmung, die politische Stabilität/Instabilität des Staates, die Migrations- und Flüchtlingsfrage sowie ein historisch-kultureller Faktor. Diese Einflussgrößen haben jedoch von Land zu Land ein unterschiedliches Ge- wicht. Im Folgenden wird dargelegt, welche Bedeutung diese Faktoren für den Aufstieg des Rechtspopulismus in Italien, Frankreich, Österreich, den Niederlanden und Deutschland haben.

\subsection{Italien}

Eindeutiger Wahlsieger bei den Parlamentswahlen in Italien im März 2018 waren die populistischen Parteien Movimento Cinque Stelle (M5S), mit einem Stimmenanteil von $32 \%$ (plus 7 Prozentpunkte), sowie die Lega (Nord) mit $17 \%$ (plus 13). Die „Sozialdemokraten“ (Partito Democratico, PD) und Berlusconis Forza Italia (FI) erreichten $18 \%$ (minus 6) bzw. $14 \%$ (minus 7 ).

Italien ist in der EU der am stärksten von (rechts-) populistischen Parteien beherrschte Staat. Von den fünf Einflussgrößen des Rechtspopulismus sind zwei - die sozioökonomische Krise und die Krise des politischen Parteiensystems - in Italien unter allen fünf Ländern am deutlichsten ausgeprägt. Italien verzeichnet eine anhaltende ökonomische Stagnationsphase, die Arbeitslosigkeit erreichte nach der Großen Finanzkrise mit 11-12\% überdurchschnittlich hohe Werte, und auch die ungelöste Bankenkrise belastet bis heute das Land (Telljohann 2016). Das tradierte Parteiensystem Italiens (pentopartito) brach unter dem Druck der Korruptionsaffären (tangentopoli) Anfang der 1990er Jahre zusammen, womit der Weg frei war für drei rechtspopulistische Parteien (Forza Italia, 
Lega Nord und Alleanza Nazionale), die unter Führung Berlusconis von 1994 bis 2011 in insgesamt vier Kabinetten das Land regierten, wenn auch mit Unterbrechungen. Es gehört zur Tragik des Landes, dass sich diese Rechtsregierungen nicht nur als unfähig erwiesen, die sozioökonomischen Probleme des Landes zu lösen, sondern auch - wie schon das vorherige Parteiensystem - an Affären (Steuerhinterziehung, Korruption, Sexskandale Berlusconis) zugrunde gingen. Von den Trümmern des Systems Berlusconis profitierte dann der M5S, der bei den Parlamentswahlen $2013 \mathrm{im}$ Abgeordnetenhaus auf Anhieb 25\% der Stimmen errang. Zwar betont der $\mathrm{M}_{5} \mathrm{~S}$, er sei weder rechts noch links, aber im Europaparlament und in Fragen der Flüchtlingskrise liegt diese Partei mit der rechtspopulistischen Lega (Nord) und den Fratelli d'Italia auf einer Linie (Caccia 2017). Die politische Instabilität kennzeichnet Italien im EU-Kontext wie kaum einen zweiten Staat. Sie ist neben der sozioökonomischen Krise der zentrale Erklärungsfaktor für die starke Bedeutung des Rechtspopulismus im Lande.

Hinzu kommt seit 2015 noch die Flüchtlingskrise, die von den populistischen Parteien genutzt wird, um Ausländerfeindlichkeit zu schüren und die Migranten als Sündenböcke für die zahlreichen Krisen des Landes abzustempeln. Da seit dem EU-Türkei-Abkommen viele Flüchtlinge den Weg über die Libyenroute nach Italien suchen, hat im Jahre 2017 die Migrationskrise einen neuen Höhepunkt erreicht. Von den EU-Staaten alleingelassen, die in der Frage eines Verteilungsmodus keinerlei Solidarität mit Italien zeigen, gewann die Kritik an der EU zusätzlich an Schwung, was die populistischen Parteien immer wieder auch in Forderungen nach einem Ausstieg aus dem Euro zuspitzten.

\subsection{Frankreich}

Mit der Machtübernahme des Front National (FN) durch Marine Le Pen im Jahre 2011 begann eine deutliche Stabilisierung der Wahlergebnisse der Partei oberhalb der 10-\%-Marke (Chwala 2015). Bei den Präsidentschaftswahlen erzielte Marine Le Pen 2012 knapp 18\%. 2017 schaffte sie es mit gut $21 \%$ in die Stichwahl gegen Emmanuel Macron, in der sie knapp $34 \%$ der Stimmen errang.

In den Wahlen zur Nationalversammlung 2012 und 2017 kam der FN jeweils auf gut $13 \%$. Besonders große Erfolge erzielte der FN bei den Europawahlen 2014, wo er mit fast $25 \%$ die erste Kraft wurde und sein Ergebnis gegenüber 2009 vervierfachte (vgl. Ivaldi 2017). Ähnlich erfolgreich für den FN verliefen die Regionalwahlen 2015, wo es ihm ebenfalls gelang, mit fast $28 \%$ im ersten Wahlgang Platz eins zu belegen. Würde in Frankreich das Verhältniswahlrecht herrschen, hätte sich der FN längst mit 20-30 \% der Mandate in den Parlamenten etabliert.

Die Wachstumsraten des Bruttoinlandsprodukts (BIP) waren in Frankreich seit der Großen Krise sehr gering. Bei sehr schwachen Zuwachsraten der Beschäftigung verharrte die Arbeitslosenrate bis 2016 bei gut 10\%. Die Versuche Sarkozys und Hollandes, die ökonomische Stagnation zu überwinden, scheiterten. Präsident Hollandes arbeitsmarktpolitische Maßnahmen zur „Umkehrung der Arbeitslosenkurve" zeigten kaum Wirkung. Viele Teile der französischen Bevölkerung sehen in der Globalisierung und der Einführung des Euro die entscheidenden Ursachen der sozioökonomischen Schwierigkeiten des Landes. Gleichzeitig machen sie die classe politique dafür verantwortlich, Frankreich unzureichend vor der Globalisierung/Europäisierung zu schützen.

Ein weiteres Problem ist die unzureichende Bewältigung der Einwanderung durch Politik und Gesellschaft. Im Hinblick auf die soziale Integration der ca. 6 Mio. Einwanderer und ihrer ca. 7 Mio. Nachfahren weist Frankreich sehr große Defizite auf. So ist die Erwerbsbeteiligung dieser Menschen mit Migrationshintergrund unterdurchschnittlich, dagegen ihre Betroffenheit von Arbeitslosigkeit überdurchschnittlich. Die Arbeitslosenrate dieser Gruppe liegt mit ca. $20 \%$ etwa doppelt so hoch wie die der Franzosen ohne Migrationsgeschichte. Die Stigmatisierung und Diskriminierung vieler, vorwiegend in den Banlieues lebender Migranten treibt etliche unter ihnen aufgrund von Enttäuschung in die Arme islamistischer und terroristischer Subkulturen, wo sie nach einer neuen Identität suchen (Kepel 2016). Gleichzeitig wächst auf der Seite der Franzosen ohne Migrationshintergrund die Kluft zwischen den Gewinnern und den Verlierern der Globalisierung/Europäisierung und bietet so dem Front National einen willkommenen Nährboden. Dieser doppelte Bruch in der französischen Gesellschaft führt dazu, dass die einen neuen Halt in der Verschärfung ihrer religiösen Identität und die anderen neue Stabilität in der Verschärfung ihrer nationalistischen Identität suchen. Terroranschläge der Dschihadisten befeuern den FN, und dessen zunehmende politische Erfolge sind Munition für die islamistische Ideologie.

\section{3 Österreich}

Unter den hier verglichenen fünf Staaten spielt nach Italien in Österreich der Rechtspopulismus die bedeutendste Rolle. Grund für diese Einschätzung ist, dass Gesellschaft und Politik in Österreich im Gefolge der Großen Finanzkrise seit 2010 eine rechtsnationalistische Verschiebung erfahren, die auch die Parteien der Großen Koalition erfasst, insbesondere die ÖVP: Nach der Großen Finanzkrise kommt es in Österreich zu einer ökonomischen Stagnation mit einer für österreichische Dimensionen hohen Arbeitslosenrate, leicht sinkenden Reallöhnen, einem Verzicht auf eine austro-keynesianische Antwort auf die Krise und stattdessen einer harten Sparpolitik. Diese sozioökonomischen Krisenerfahrungen werden in den Unter- und Mittelschichten begleitet von einer Angst vor der Globalisierung und der Europäisierung. Sie artikulieren sich auch in einer zunehmenden Kritik an den beiden Konkordanz- 
parteien ÖVP und SPÖ, die Österreich seit 1945 mehr als 50 Jahre lang in Großen Koalitionen regiert haben. Die Auffassung, dass diese Große Koalition die ökonomischen Probleme des Landes nicht bewältigen könne, war zuletzt stark verbreitet.

Wie in den anderen untersuchten Ländern greift die rechtspopulistische Partei in Österreich, die FPÖ, diese sozialökonomischen und politischen Probleme auf, um sie in Form eines Anti-Globalisierungs-, Anti-EU-, Anti-Systemparteien- und Anti-Ausländer-Diskurses zu artikulieren. Zusätzlich wird, wie in den anderen Ländern, auch in Österreich die Flüchtlingskrise seit 2015 von den „Freiheitlichen“ genutzt, um die Migranten als Verursacher der sozialen Krise abzustempeln. Der entscheidende Unterschied gegenüber den vier anderen Staaten besteht jedoch darin, dass sich die Parteien der Großen Koalition, vor allem die ÖVP, aber zeitverzögert auch Teile der SPÖ, diesem Anti-Migrations-Diskurs immer stärker angepasst haben, ja, im Falle der Kurz-Partei, sogar versucht haben, die FPÖ rechts zu überholen. Obwohl die von Österreich selbst gesetzte Obergrenze von 35000 Flüchtlingen für 2017 mit ca. 20000 weit unterschritten wird, überschlagen sich bei FPÖ und ÖVP die Forderungen nach Abschottung, Abschiebung und Unterbringung von Flüchtlingen auf Inseln im Mittelmeer oder in Lagern in Afrika und im Nahen Osten.

Wir beobachten in Österreich einen Rechtsruck also nicht nur deswegen, weil die FPÖ gestärkt wird, sondern auch aufgrund einer zunehmenden nationalistischen Überlagerung aller politischen, nicht zuletzt sozialer Fragen auch in den Parteien der Großen Koalition. Es geht um eine Rechtsverschiebung quasi großer Teile des Landes (Pelinka 2002, 2017).

\subsection{Niederlande}

Auch in den Niederlanden fällt das Wirtschaftswachstum als Folge der großen Finanz- und Wirtschaftskrise 2008ff. zunächst schwach aus. Zurückgeworfen wurde das Land vor allem in den beiden Rezessionsjahren der Eurozone, 2012 und 2013, die das Ergebnis des harten Euro-Austeritätsregimes waren. Seitdem erholt sich das Land aber sichtlich: Die Zuwachsraten des BIP ziehen deutlich an. Die Reallöhne pro Kopf sind seit 2011 moderat angestiegen (vgl. Busch et al. 2018, S.110ff.).

Im Bewusstsein der niederländischen Bürgerinnen und Bürger ist diese Verbesserung der makroökonomischen Daten jedoch dadurch negativ überlagert worden, dass kein anderes Land in der Vergleichsgruppe so hart gespart hat wie die Niederlande. Das Haushaltsdefizit ist in den Niederlanden von gut $5 \%$ im Jahr 2010 in einen Haushaltüberschuss in Höhe von 0,4 \% im Jahr 2016 transformiert worden. Die Bürgerinnen und Bürger in den Niederlanden haben dadurch einen starken Rückgang wohlfahrtsstaatlicher Leistungen erfahren, und zwar in den Bereichen Gesundheit, Pflege, Renten und Bildung. Darüber hinaus ist in den Niederlanden seit der Großen Finanzkrise die soziale Ungleichheit angestiegen: die Spreizung in der Einkommensverteilung ist gewachsen, das Armutsproblem hat sich vergrößert, und auf dem Arbeitsmarkt der Niederlande spielt nach wie vor die Prekarisierung eine wesentlich größere Rolle als im Durchschnitt der EU. Nicht nur die Unterschichten, auch die Mittelschichten fühlen sich vom sozialen Abstieg bedroht.

Vor diesem Hintergrund gibt die rechtspopulistische Partei PVV von Geert Wilders im Jahre 2012 ihre Tolerierungspolitik für das erste Kabinett Rutte auf, das seit 2010 zwischen der rechtsliberalen VVD und der christlichen CDA als Minderheitsregierung gebildet wurde. Die PVV profiliert sich damit als soziale Partei, welche die vom Euroregime diktierte Sparpolitik ablehnt.

Da auch das zweite Kabinett Rutte (2012-2017), eine Große Koalition aus VVD und sozialdemokratischer PvdA, die harte Sparpolitik fortsetzt, kann sich die rechtspopulistische Partei von Geert Wilders weiterhin als Partei darstellen, die den holländischen Wohlfahrtsstaat verteidigen will. Sie verbindet dies - wie die Rechtspopulisten in den anderen Ländern - mit einem Anti-Migrationsdiskurs und versucht insbesondere, die Muslime in den Niederlanden zu den Sündenböcken für die sozialen Einsparungen und die wachsende soziale Ungleichheit zu machen.

Im Wahlkampf 2017 zeigte sich, dass diese Strategie von Geert Wilders auf fruchtbaren Boden fiel. Lange Zeit sah es so aus, dass die PVV stärkste Partei werden könnte. Erst der deutliche Rechtsschwenk von Rutte, der in seinem Brief an die Holländer die Migranten aufforderte, sich „normal“ zu verhalten oder das Land zu verlassen, verhinderte den Erfolg von Geert Wilders, dessen PVV nach der VVD Ruttes „nur“ den zweiten Platz erreichte. Die Sozialdemokraten, die PvdA, zahlte für ihre Unterstützung der Sparpolitik in der Großen Koalition des zweiten Kabinetts Rutte einen hohen Preis: Sie schrumpfte bei den Wahlen 2017 um fast 20 Prozentpunkte und wurde auf 5,6\% dezimiert.

\subsection{Deutschland}

Unter den fünf untersuchten Staaten erscheint Deutschland als das mit Abstand (noch) stabilste Land. Deutschland verzeichnet nach der Großen Krise die höchsten Wachstumsraten und den stärksten Abbau der Arbeitslosigkeit. Auch die Reallöhne entwickeln sich wieder positiv. Problematisch - und ein zentraler Erklärungsfaktor für das Erstarken des Rechtspopulismus - ist allerdings die Entwicklung der Verteilungsverhältnisse und die zunehmende Wahrnehmung einer sozialökonomischen Ungleichheit. Die unteren 40\% der Einkommensbezieher verzeichnen in den letzten 20 Jahren kaum Zuwächse. Die Angst der unteren Schichten bis hinein in die unteren Mittelschichten vor einem sozialen Abstieg aufgrund der Globalisierungsfolgen ist ausgeprägt. Hinzu kommt, dass in den neuen Bundesländern ein starker Teil der Bevölke- 
rung sich im Vergleich mit den Westdeutschen als Bürgerinnen und Bürger zweiter Klasse wahrnimmt.

Was die politische Stabilität des Landes anbelangt, so dominierten zwar bis vor Kurzem immer noch die beiden Parteien der Großen Koalition die Szenerie, aber ihre Verluste bei der letzten Bundestagswahl 2017 waren gravierend (zusammen 14 Prozentpunkte), und beide Parteien liegen zusammen nur noch knapp über der Marke von $50 \%$. Auch in Deutschland wächst bei einem Teil der Bevölkerung der Eindruck, dass die großen Volksparteien die Probleme des Landes immer weniger zufriedenstellend lösen können. Bei den Landtagswahlen in Bayern und Hessen 2018 erreichten die Verluste von CDU/CSU und SPD jeweils zweistellige Größen. In den Meinungsumfragen zur Bundestagswahl rutschte die CDU/CSU zwischenzeitlich auf ca. $25 \%$ ab, und die SPD lag zeitweise bei $14 \%$. Befeuert wurde die AfD, die 2017 mit 12,7\% erstmals in den Bundestag einzog (vgl. Funke/Mudra 2018), von dem vierten Erklärungsfaktor, der Flüchtlingskrise. Dominierte in Deutschland noch 2015 die Willkommenskultur, ist diese längst der wachsenden Skepsis einer Mehrheit der Bürgerinnen und Bürger vor einem Zustrom an Zuwanderern gewichen. Die Parteien der Mitte reagieren darauf mit einer Politik der Abschottung und des Abschiebens (EU-Türkei-Abkommen, mehr Staaten werden zu sicheren Drittstaaten erklärt) und übernehmen immer stärker die Rhetorik der AfD in der Flüchtlingsfrage. Selbst vor der Unterstützung der völkerrechtswidrigen und menschenverachtenden Abschirmung der Libyenroute macht dieser Kurswechsel nicht Halt.

Neben der ökonomischen und der politischen Stabilität ist der historisch-kulturelle Erklärungsfaktor für die im Vergleich zu den anderen Staaten immer noch stärkere Eindämmung des Rechtspopulismus in Deutschland verantwortlich. Im Unterschied zu Frankreich und vor allem Österreich ist der Faschismus in der BRD seit der Studentenbewegung und der demokratischen Reformphase, die mit Willy Brandt einsetzte, kritisch aufgearbeitet worden. Nach wie vor stellt es in Deutschland eine hohe Barriere dar, eine Partei zu wählen, welche die Gräueltaten der NSZeit relativiert.

\section{Aufschwung des Rechtspopulismus, wachsende politische Instabilität und die Folgen für die Wahlen zum Europaparlament}

Die Länderanalysen haben nicht nur die zunehmende Erstarkung des Rechtspopulismus gezeigt, sie offenbaren darüber hinaus eine wachsende Instabilität im Parteiensystem in den untersuchten Ländern. Am markantesten ist dieser Trend in Italien und den Niederlanden, aber auch in
Frankreich und Österreich stehen die Systeme vor einem Umbruch; und selbst Deutschland wird tendenziell von dieser Entwicklung erfasst.

Italien hat in den 1990er Jahren den Zusammenbruch des Pentopartito erlebt, sah sich dann mit dem Aufschwung rechtskonservativer und rechtspopulistischer Parteien konfrontiert (Forza Italia, Lega Nord, Alleanza Nazionale), die von 1994 bis 2011 mit Unterbrechungen unter Berlusconi vier Kabinette stellten, aber die sozialökonomische Misere des Landes trotz großspuriger Versprechungen nicht überwinden, sondern eher verschärfen sollten. Von diesem doppelten Zusammenbruch des politischen Systems profitierte seit 2013 verstärkt der Movimento Cinque Stelle, der nach den Parlamentswahlen im März 2018 mit der Lega die Macht übernommen hat. In den jüngsten Wahlumfragen läuft inzwischen die Lega unter Salvini dem $\mathrm{M}_{5} \mathrm{~S}$ den Rang ab und erzielt dabei $33 \%$ der Stimmen, während der $\mathrm{M}_{5} \mathrm{~S}$ bei $30 \%$ rangiert. Die bei den letzten Wahlen dezimierte Linke verzettelt sich derweil in internen Machtkämpfen und schaut dem scharfen Rechtsruck des Landes in Fragen der Europa- und der Flüchtlingspolitik mehr oder weniger ohnmächtig zu. Zwar dominieren die populistischen Parteien gemeinsam mit der rechtsnationalistischen Fratelli d'Italia zwei Drittel der Wählerschaft, zu mehr politischer Stabilität trägt dies jedoch nicht bei. Im Gegenteil: Der Konfrontationskurs, den die neue Regierung Italiens zwischenzeitlich mit ihrer Haushaltspolitik gegenüber der EU beschritten hat, hat die ökonomischen Probleme des Landes eher verschärft. Darüber hinaus gibt es zwischen den beiden Regierungsparteien erhebliche Auseinandersetzungen darüber, wie die großen Wahlversprechen, die sich als nicht finanzierbar erweisen, reduziert werden sollen.

In den Niederlanden kontrollieren die beiden ehedem stärksten Parteien, CDA und PvdA, längst nicht mehr die Parlamente. Zusammen mit der rechtsliberalen VVD errangen sie noch in den 1990er Jahren mehr als 80\% der Stimmen. Seitdem ist deren Anteil im Trend gesunken und lag in den ersten 20ooer Jahren noch bei ca. $60 \%$. Bei den Wahlen 2010 sank der Anteil von CDA und PvdA, die noch 2003 jeweils weit mehr als $25 \%$ der Stimmen erhielten, unter $20 \%$, und 2017 fiel die PvdA auf 5,7\% und die CDA auf 12,5\%, während die Rutte-Partei VVD 21,3\% und die Wilders-Partei PVV 13,1\% erreichten. Gegenüber den 1990er und den ersten 2oooer Jahren ist dieser Absturz des Anteils der drei größten Parteien auf nicht einmal 40\% gewaltig. Diese zunehmende Zersplitterung der Parteienlandschaft hat die Regierungsbildung 2017 sehr erschwert. Erst nach einem halben Jahr konnte Rutte eine Vier-Parteien-Regierung konstituieren.

In Frankreich haben die Wahlen 2017 einen Zusammenbruch des bipolaren Parteiensystems gebracht, das aus der Quadrille bipolaire entstand und seit den 197oer Jahren die V. Republik mit der PS und den Republikanern (LR, vorher UMP) als Links-Rechts-Alternative die Politik beherrschte. Macrons La République en Marche (LREM) 
ist eine Partei der Mitte, die selbst ihr Spitzenpersonal zum Teil aus den Parteien PS und LR rekrutiert. Nach der Dezimierung der PS (7,4\%, minus 22 Prozentpunkte) und der Kooperation eines Reformflügels der Republikaner (Juppé-Flügel) mit Macron bilden vor allem Mélenchons „La France Insoumise" und Le Pens Rassemblement National die Opposition, also extreme Parteien am linken und rechten Rand. Wie sich die Wahl des neuen Vorsitzenden Laurent Wauquiez, der von sich behauptet, er sei vor allem „rechts“, auf die Entwicklung der gespaltenen Republikaner auswirkt, bleibt abzuwarten.

Stabil ist das neue System nicht, bildet doch Macrons LREM selbst für französische Verhältnisse nur eine schwache Partei mit einem geringen organisatorischen Unterbau. Instabil ist dieses System auch, weil der bereits vor dem Erscheinen der Gilets jaunes geschwächte Macron bei einem Scheitern seines Reformprogramms und bei geringen sozioökonomischen Erfolgen in den nächsten Präsidentschaftswahlen einem Gegenkandidaten der extremen Linken oder Rechten gegenüberstünde, der, gleich welcher politischen Couleur, nicht ohne beachtliche Erfolgschancen wäre. Die Erfolge der Gilets jaunes im Kampf gegen Macrons Politik Ende 2018 haben diese strukturelle Instabilität noch verschärft.

Auch in Österreich nimmt die politische Instabilität mit dem Scheitern der Großen Koalition und der Bildung einer ÖVP-FPÖ-Regierung nach den Wahlen $2017 \mathrm{zu}$. Aufgrund des Rechtsrucks des Landes - und das ist die Befürchtung der Gewerkschafen und der SPÖ - könnte die neue Koalition das System der Arbeiterkammern abschaffen. Dieses bildet aber den administrativen, wissenschaftlichen und ideologischen Unterbau der Gewerkschaften, unter deren Schwächung auch die SPÖ im hohen Maße leiden würde. Es hat den Anschein, dass aufgrund dieser möglichen Entwicklungen die Zeit der Großen Koalitionen und damit der Konkordanzdemokratie erst einmal vorbei ist.

In Deutschland zerbröckelt - wie oben dargestellt die Macht der Parteien der Großen Koalition, während die Grünen und die AfD in Umfragen deutlich zulegen. Nach der Wahl von Annegret Kramp-Karrenbauer zur neuen CDU-Vorsitzenden ist unklar, wie lange Angela Merkel noch Bundeskanzlerin bleiben wird. Ein Ende der Großen Koalition, Neuwahlen und eine Jamaika-Koalitionsregierung könnten die Folge sein. Der Machtverlust Merkels und die Rechtsverschiebung innerhalb der CDU/ CSU sind im hohen Maße auch Resultat des Aufschwungs der Rechtspopulisten.

Die zunehmende politische Instabilität, die großen Verluste der konservativen und sozialdemokratischen „Volks“parteien und die Stärkung des Rechtspopulismus werden auch erhebliche Auswirkungen auf die Wahlen zum Europäischen Parlament im Mai 2019 haben (vgl. Körner 2018). Die bislang hier herrschende große Koalition aus EVP und S\&D, die für die Stabilität des Gesetzgebungsprozesses von Bedeutung war, wird aller Voraussicht nach die absolute Mehrheit der Mandate verlieren. Die EVP könnte $25 \%$ der Sitze erreichen (minus 4 Prozentpunkte), die S\&D verliert möglicherweise noch mehr und erzielt $19 \%$ (minus 6). Je nach Orientierung von Macrons LREM würde die liberale ALDE 10-13\% der Mandate bekommen. Die größten Zugewinne würden die verschiedenen Fraktionen der Europagegner und Europaskeptiker verbuchen (ENF, EFDD, ECR), die von jetzt $18 \%$ auf gut $25 \%$ ansteigen könnten.

Damit würde der von Salvini, di Maio und Le Pen angekündigte Machtwechsel in Brüssel zwar ausbleiben, der Gesetzgebungsprozess würde aber schwieriger werden. Die Koalitionsbildung für Gesetze müsste jetzt neben der EVP und den S\&D auch die Liberalen und/oder die Grünen einbeziehen (vgl. Wientzek 2018).

\section{Der Rechtspopulismus blockiert die Lösung von Integrationskonflikten}

EU und Eurozone kämpfen zurzeit an verschiedenen Fronten mit Problemen, ohne diese lösen zu können. Im Gegenteil, die Blockaden scheinen größer zu werden. $\mathrm{Zu}$ den wichtigsten Konflikten zählen:

- Der Austritt Großbritannien aus der EU, der im Frühjahr 2019 möglicherweise ungeregelt - ohne Abkommen - stattfinden wird;

- die schwelende Flüchtlingskrise, in der es der EU trotz europäischer Kompetenz - nicht gelingt, einen obligatorischen Verteilungsmechanismus durchzusetzen;

- die seit dem Höhepunkt der Eurokrise 2011/2012 laufende Debatte über notwendige Reformen zur Stabilisierung der Eurozone, die auf dem Gipfel im Dezember 2018 einen großen Rückschlag verzeichnete;

- der Bruch von Demokratie und Rechtsstaatlichkeit in Polen, Ungarn und Rumänien, ohne dass diese Staaten von der EU auf dem Weg in „illiberale Demokratien“ zur Umkehr gezwungen werden.

In ihrer Geschichte hat die EU immer wieder Rückschläge hinnehmen müssen. Immer ist es aber gelungen, selbst nach gravierenden Einschnitten, wie der „Politik des leeren Stuhls“ durch de Gaulle Mitte der 1960 Jahre oder dem Scheitern des ersten WWU-Plans Ende der 1970er Jahre, mit entscheidenden Vertiefungsschritten auf den Integrationspfad zurückzukehren. Die Verabschiedung der „Einheitlichen Europäischen Akte“ 1987 und die Verträge von Maastricht (1993), Amsterdam (1999) und Nizza (2003) brachten der EU ein Golden Age der Integration mit entscheidenden politischen und ökonomischen Fortschritten. Das Scheitern des Verfassungsvertrages der EU im Jahre 2005 beendete jedoch diese Aufschwungsphase 
des Integrationsprozesses, und seitdem will kein neuer Durchbruch gelingen. Die EU befindet sich vielmehr auf verschiedenen Feldern in einer Sackgasse.

Die entscheidende Ursache für diese Stagnationsperiode der Integration ist im wachsenden Trend zum Rechtspopulismus zu sehen, der vor allem durch die nach der Großen Finanzkrise 2008/2009 einsetzende Austeritätspolitik einen Aufschwung erfahren hat. Die Politik der Re-Nationalisierung hat den Brexit hervorgerufen, verhindert eine solidarische Verteilungspolitik im Rahmen der Flüchtlingskrise, lässt keine entscheidenden Fortschritte bei der Reform der Eurozone zu und ist die wichtigste Antriebskraft für die polnische PIS und die ungarische Fidesz.

\subsection{Re-Nationalisierung und Flüchtlingspolitik}

Der Anstieg des Rechtspopulismus hat in der EU zu einer Verschärfung der Migrationskonflikte geführt. Aufgrund einer massiven Politik der Abschottung - Verschärfung des Asylrechts, verstärkte Abschiebungen, EU-Türkei-Abkommen, Ausbau von Frontex und Stärkung der Zusammenarbeit mit Libyen - ist es zwar zu einer deutlichen Reduktion der Flüchtlingszahlen gekommen, aber die entscheidenden Fragen der ungleichen Belastung der EU-Staaten durch Flüchtlinge und der Umsetzung der Verteilungsbeschlüsse der EU zur Entlastung von Italien und Griechenland sind bis heute nicht gelöst. Die Visegrad-Staaten verweigern es, sich an der Aufnahme von Flüchtlingen zu beteiligen, und vor allem Italien kritisiert, von der EU letztlich allein gelassen zu werden. Zu Konflikten kommt es auch aufgrund der Sekundärmigration und der Durchsetzung des Dublin-Systems.

Der Gipfel des Europäischen Rats im Juni 2018 hat Maßnahmen beschlossen, die eine weitere Verschärfung der Abschottung (Stärkung von Frontex und der libyschen Küstenwache), den Aufbau von „Ausschiffungsplattformen" in Drittstaaten (Kasernierung der auf der Flucht aufgegriffenen Migranten zur Klärung ihres Status) und den Aufbau von „internen Zentren“ in den Mitgliedstaaten (Kasernierung von Flüchtlingen zur Klärung ihres Status und Einleitung von Umsiedlungs- und Neuansiedlungsmaßnahmen „unbeschadet der Dublin-Reform“) beinhalten (Europäischer Rat 2018a).

Auch wenn sich die EU mit diesen Beschlüssen immer mehr auf den Pfad einer rechtspopulistischen Flüchtlingspolitik begibt, deren Konformität mit dem Völkerrecht fraglich ist und die gegen Menschenrechte verstößt, zu deren Einhaltung sich die EU in vielen Rechtstexten verpflichtet hat, wird diese Politik kaum zu einer Lösung der Konflikte zwischen den Mitgliedstaaten führen. Denn: Weder ist geklärt, welche Staaten in Nordafrika zum Aufbau von "Ausschiffungsplattformen" bereit sind, ${ }^{1}$ noch, welche Staaten die von dort in die EU zu verbringenden Flüchtlinge aufnehmen wollen. Auch ist nicht geregelt, welche Staaten die „internen Zentren“ aufbauen sollen, und welche bereit sind, sich an den von dort ausgehenden Umsiedlungsmaßnahmen zu beteiligen. All diese Unklarheiten bis hin zu Verweigerungen einzelner EU-Mitgliedsländer sind Folge des auf dem EU-Gipfel vom Juni 2018 vereinbarten „Prinzips der Freiwilligkeit“, das die EU akzeptiert hat, um überhaupt zu Beschlüssen kommen zu können. Jedoch: Mit der Anerkennung des Prinzips der Freiwilligkeit hat die EU das vertragswidrige Verhalten der Visegrad-Staaten legitimiert und sich letztlich ihrer Handlungsmöglichkeiten im Falle von Konflikten zwischen den Mitgliedstaaten beraubt. Es wird weiterhin dabei bleiben, dass Staaten ungleich belastet sind und dass sich nicht alle Staaten an der Verteilung von Migranten aus Nordafrika oder aus den ,internen Zentren“ einzelner Staaten beteiligen werden. Damit bleiben aufgrund der Re-Nationalisierungstendenz die grundlegenden Probleme zwischen den Mitgliedstaaten erhalten, die der Juni-Gipfel ursprünglich vorgab, lösen zu wollen.

Auch die EU-Gipfel vom Oktober und Dezember 2018 haben bei den strittigen Fragen keinen Durchbruch gebracht. Einigkeit besteht bei einem personellen Ausbau von Frontex, wenn auch im wesentlich langsameren Tempo als von der Europäischen Kommission vorgeschlagen. An den Fragen des Gemeinsamen Europäischen Asylrechts (sieben Gesetzesvorschläge), der Einrichtung einer Asylagentur und einer gemeinsamen Rückführungsrichtlinie soll weiter gearbeitet werden (Europäischer Rat 2018b). Der Versuch der Kommission, die Beratungen über ein Gemeinsames Asylrecht zu beschleunigen, indem aus dem Gesetzespaket die Frage eines gemeinsamen Verteilungssystems herausgenommen wird, scheiterte trotz großer Unterstützung aus Österreich und den Visegrad-Staaten am Widerstand Deutschlands.

\subsection{Das (bisherige) Scheitern der WWU-Reformen}

Seitdem in den Jahren nach 2010 in der Eurokrise die Defizite der Europäischen Wirtschafts- und Währungsunion (WWU) überdeutlich wurden, diskutiert die EU verstärkt über eine Reform der Konstruktion des Euro.

2012 legte die Barroso-Kommission eine Blaupause für die Reform der WWU vor, die folgende wichtige Elemente beinhaltete: Einführung einer Wirtschaftsregierung, welche die Kompetenz für eine antizyklische Fiskalpolitik erhalten sollte, Eurobonds und ein Schuldentilgungsfonds für eine gemeinsame europäische Schuldenpolitik. Dem EP wurde in diesen Plänen die Kompetenz zur demokratischen Kontrolle dieser Wirtschaftsregierung übertragen. Spätestens die in den Europawahlen 2014 sichtbar werdende Re-Nationalisierungstendenz machte jedoch deutlich, dass diese sinnvollen, aber weitgehenden Reformpläne auf

Bis Dezember 2018 hat sich kein Staat in Afrika bereit erklärt, solche „Ausschiffungsplattformen” aufzubauen! 
massiven Widerstand stoßen würden. Die Reformdebatte versandete daraufhin und wurde erst 2017 durch neue Vorschläge der Europäischen Kommission und des neuen Präsidenten Frankreichs, Emmanuel Macron, wiederbelebt.

Die Pläne der Kommission aus dem Frühjahr und dem Herbst 2017, die nicht im Entferntesten so weit gingen wie die oben erwähnten Barroso-Pläne, beinhalteten im Wesentlichen einen etwas vergrößerten EU-Haushalt, der auch Mittel für eine Bekämpfung von asymmetrischen Krisen sowie zur Förderung von (neoliberalen) Reformen in einzelnen EU-Staaten bekommen sollte, ohne freilich Transferleistungen von der EU für jene Mitgliedstaaten vorzusehen.

Emmanuel Macrons Vorschläge waren zwar weitergehend, aber noch nicht sehr detailliert. Macron ging es im Wesentlichen um einen deutlich größeren EU-Haushalt und einen eigenständigen Haushalt für die Eurozone, der Krisen bekämpfen und Investitionen anstoßen sollte.

Im Verlaufe der Verhandlungen des Jahres 2018 scheiterte der überwiegende Teil der Reformvorschläge sowohl der Europäischen Kommission als auch Emmanuel Macrons. Dieses Scheitern ist einerseits auf die nur zögerliche Unterstützung der Ideen Macrons durch die Regierung Merkel, andererseits den Widerstand der „Hanseatischen Liga“ zurückzuführen. Merkels Zurückhaltung hatte ihre Ursache vor allem in der Ablehnung der Pläne für einen größeren EU-Haushalt und einen eigenständigen Eurozonenhaushalt mit Stabilisierungsfunktion in der CDU/ CSU-Bundestagsfraktion. Und diese Verweigerung von mehr Mitteln für die EU und eines eigenständigen Eurozonenhaushalts wurde seit dem Frühjahr 2018 von einer als „Hanseatische Liga“ bezeichneten Staatengruppe noch wesentlich strikter vorgetragen, die zunächst acht EU-Staaten (Niederlande, drei skandinavische, drei baltische Staaten sowie Irland) und später zwölf Staaten (zusätzlich Belgien, Luxemburg, Malta und Österreich) umfasste.

In der „Hanseatischen Liga“, die sich gegen eine Vertiefung des europäischen Integrationsprozesses zur Wehr setzt, befinden sich zahlreiche Staaten (Belgien, Dänemark, Schweden, Finnland, Niederlande, Österreich), in denen die rechtspopulistischen Parteien ein solches Gewicht haben, dass sie das politische Klima des Landes stark beeinflussen. In einigen dieser Staaten (Belgien, Dänemark, Österreich) sind sie inzwischen auch an Koalitionsregierungen beteiligt.

Auf dem Juni-Gipfel 2018 wurden alle Entscheidungen über eine Reform vertagt, aber auch die Erfolgsaussichten des Dezember-Gipfels wurden im Verlaufe von Sommer und Herbst nicht besser.

Die Verstimmung Frankreichs über die mangelnde Unterstützung aus Deutschland wurde von Monat zu Monat stärker.

Frankreich und Deutschland präsentierten zwar schließlich im November einen Vorschlag für einen Eurozonenhaushalt, der aber erhebliche Mängel hatte. Weder waren der Umfang dieses Haushalts, noch seine Finanzierung, noch seine Struktur geregelt. Seine Aufgaben sollten sich sehr allgemein auf die Bereiche Konvergenz, Wettbewerbsfähigkeit und Stabilisierung erstrecken. Allgemein wurde dieser Vorschlag als Initiative bewertet, die der Gesichtswahrung des französischen Präsidenten dienen sollte.

Doch auch dieses bescheidene Vorhaben war von vornherein ohne Erfolgschancen, artikulierten doch die Niederlande, Österreich und Italien unmittelbar Vorbehalte. Der Wortführer der „Hanseatischen Liga“, der niederländische Finanzminister Hoekstra, erklärte, er könne dem Vorschlag keinen Sinn abgewinnen.

Auf der Sitzung der Finanzminister der Eurogruppe am 4.12.18 wurde der deutsch-französische Vorschlag auseinandergenommen. Im Bericht an den Europäischen Rat heißt es, über einen Eurozonenhaushalt zur Verbesserung von Konvergenz und Wettbewerbsfähigkeit könne weiter verhandelt werden, über einen derartigen Haushalt zur Stabilisierung der Eurozone jedoch sei unter den Mitgliedstaaten keine Einigkeit herzustellen (Eurogroup 2018).

Der Euro-Gipfel am 14.12.18 beerdigte diese Stabilisierungsfunktion dann endgültig. In der Erklärung des Gipfels wird sie nicht einmal mehr erwähnt (Euro-Gipfel 2018). Dagegen soll am Haushaltsinstrument für Konvergenz und Wettbewerbsfähigkeit weiter gearbeitet werden. Auch in Bezug auf dieses Instrument bleibt aber letztlich alles offen, da über seinen finanziellen Umfang im Rahmen des Mittelfristigen Finanzrahmens (MFR) zu einem späteren Zeitpunkt, und zwar mit Einstimmigkeit, zu entscheiden ist.

Weitere Beschlüsse des Euro-Gipfels beziehen sich auf den ESM, der zwar nicht - wie von der Kommission gewünscht - in einen Europäischen Währungsfonds umgewandelt werden, aber gewisse Erweiterungen erfahren soll. Doch auch hier unterliegen die Details weiteren Verhandlungen in den Jahren 2019 und 2020. Als zentrales Ergebnis des Dezember-Gipfels ist festzuhalten, dass die makroökonomische Stabilisierung der Eurozone, das Kernelement aller WWU-Reformvorschläge, seit der Barroso-Blaupause von 2011, zu Grabe getragen wurde.

Man könnte meinen, dass es sich bei diesem Scheitern einer Reform der Eurozone um die bekannten Verzögerungen handelt, die sich auch in der Vergangenheit immer dann einstellten, wenn es um wichtige Vertiefungen des Integrationsprozesses ging. Diese Interpretation verkennt, dass sich sehr viele EU-Staaten seit einigen Jahren in einem Prozess der politischen Rechtsverschiebung und der Stärkung der rechtspopulistischen Ränder befinden. Damit einher geht eine Zunahme des Nationalismus und der Kritik an der EU, die statt einer weiteren Vertiefung eine Rückbildung des Integrationsprozesses fordert. Solange dieses politische Klima nicht nur in einigen, sondern in der Mehrheit der EU-Staaten dominiert, wird - wie oben gezeigt - die Handlungsunfähigkeit der EU anhalten und der Integrationsprozess zunehmend gefährdet. 


\section{LITERATUR}

Busch, K./ Bischoff, J./ Funke, H. (2018): Rechtspopulistische Zerstörung Europas - Wachsende politische Instabilität und die Möglichkeiten einer Kehrtwende, Hamburg

Caccia, B. (2017): Drei Populismen und kein ,Volk'. Politische Konstellationen in Italien, in: Luxemburg. Gesellschaftsanalyse und Linke Praxis, Berlin

Chwala, S. (2015): Der Front National. Geschichte, Programm, Politik und Wähler, Köln

Euro-Gipfel (2018): Tagung des Euro-Gipfels - Erklärung, https://www.consilium europa.eu/media/37599/14-eurosummit-statement-de.pdf, Brüssel, Dezember Eurogroup (2018): Report to Leaders on EMU deepening, https://www.consi lium.europa.eu/de/press/press-releases/2018/12/o4/eurogroup-report-to-leaderson-emu-deepening, Brussels, Dezember

Europäischer Rat (2018a): Tagung des Europäischen Rates - Schlussfolgerungen, http://data.consilium.europa.eu/doc/document/ST-9-2018-INIT/ de/pdf, Brüssel, Juni

Europäischer Rat (2018b): Tagung des Europäischen Rates Schlussfolgerungen, http://data.consilium.europa.eu/doc/document/ST-17-2018-INIT/ de/pdf, Brüssel, Dezember

Funke, H. / Mudra, Ch. (2018): Gäriger Haufen. Die AfD: Ressentiments, Regime wechsel und völkische Radikale, Hamburg

Ivaldi, G. (2017): Europa in der Konfrontation mit populistischen und rechtsradikalen Parteien, in: Hentges, G. / Nottbohm, K./ Platzer, H.-W. (Hrsg.): Europäische Identität in der Krise? Europäische Identitätsforschung und Rechtspopulismusforschung im Dialog, Wiesbaden, S.121-148
Keppel, G. (2016): Terror in Frankreich - Der neue Dschihad in Europa, München Körner, K. (2018): Europawahl 2019 - Die nächste ,Schlacht um Europa'?, in: Deutsche Bank Research, Frankfurt a. M.

Pelinka, A. (2002): Die FPÖ im internationalen Vergleich. Zwischen Rechtspopulismus, Deutschnationalismus und Österreich-Patriotismus, in: conflict \& communication online 1 (1), Köln

Pelinka, A. (2017): Türkis ist das neue Blau. Die FPÖ wird die Herbstwahl zwar nicht gewinnen, ihre Ideologie hat sich aber durchgesetzt, in: Zeit online vom 08. 09. 2017, Hamburg

Telljohann, V. (2016): Italien: Marginalisierung des Tripartismus, in: Bsirske, F. / Busch, K. / Höbel, O. / Knerler, R. / Scholz, D. (Hrsg.): Gewerkschaften in der Eurokrise - Nationaler Anpassungsdruck und europäische Strategien, Hamburg, S. $139-156$

Wientzek, O. (2018): EVP-Parteienbarometer - Oktober/November 2018, Konrad-Adenauer-Stiftung, Berlin

\section{AUTOR}

KLAUS BUSCH, Professor (i. R.) für Europäische Studien, Universität Osnabrück. Forschungsschwerpunkte: Wirtschafts- und Währungsunion, Soziales Europa, Rechtspopulismus in der EU.

@busch@uos.de 